\title{
Research Paper: \\ The Effect of Selected Exercise Group on Motor Skills and Cognitive Functions in Children With Autism Spectrum Disorders
}

Mahboubeh Ghayour Najafabadi ${ }^{1}$ (D), Amir Sobh-Rhakhshankhah ${ }^{2^{*}}$ (D), Sepideh Niyazi ${ }^{3}$ (D)

1. Department of Motor Behavior, Faculty of Physical Education and Sport Science, University of Tehran, Tehran, Iran.

2. Sepehr Heart Center of Baharloo Hospital, Tehran University of Medical Sciences, Tehran, Iran.

3. Shariatee Hospital, Tehran University of Medical Sciences, Tehran, Iran.

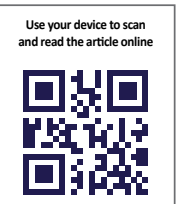

Citation Ghayour Najafabadi M, Sobh-Rhakhshankhah A, Niyazi S. The Effect of Selected Exercise Group on Motor Skills and Cognitive Functions in Children With Autism Spectrum Disorders. Journal of Exercise Science and Medicine (JESM). 2019; 11(2):105-112. http://dx.doi.org/10.32598/JESM.11.2.5

doi' http://dx.doi.org/10.32598/JESM.11.2.5

\section{(c) (i) (5)}

Article info:

Received: 10 Feb 2019

Accepted: 13 May 2019

Available Online: 01 Jul 2019

Keywords:

Sports, Play and acyive Recreation for Kids (SPARK) , Autism spectrum disorder, Executive function, Motor skill

\section{ABSTRACT}

Introduction: The present study aimed to determine the effects of a selected group exercise on motor skills and cognitive function in children with Autism Spectrum Disorders (ASDs).

Methods: Forty children with ASD aged 5-12 years participated in this quasi-experimental study The Bruininks-Oseretsky Test of Motor Proficiency (BOTMP), and the Wisconsin Card Sorting Test (WCST) were performed in 3 assessment stages of baseline, pretest, and posttest in Sports, Play, and Active Recreation for Kids (SPARK). The repeated-measures Analysis of Variance (ANOVA) and Analysis of Covariance (ANCOVA) were used for analyzing the collected data.

Results: The current research results demonstrated that the selected exercises presented positive effects on motor skills $(P<0.05)$ in the studied subjects; however, they indicated no significant effects on their cognitive function ( $P>0.05$ ).

Conclusion: According to the present study results, the SPARK's training is effective on children with ASDs and severe mobility limitations; however, it presented no cognitive function improvement in the study participants.

\section{Introduction}

he Centers for Disease Control and Pre$\mathrm{T}$ vention $(C D C)$ has recently estimated that 1 in 59 children in multiple states in the united states has been diagnosed with Autism Spectrum Disorders (ASDs) [1]. According to the Diagnostic and Sta- tistical Manual of Mental Disorders, Fifth Edition, Text Revision (DSM-V-TR), the core characteristics of ASDs include impairments in language and communication skills, as well as the presence of restricted and repetitive behaviors and interests [2]. These characteristics are associated with challenging and disruptive behaviors that prevent children with ASD from usual physical activities; therefore, they deprive them of learning

* Corresponding Author:

Amir Sobh-Rhakhshankhah, PhD.

Address: Sepehr Heart center of Baharloo hospital, Tehran University of Medical Sciences, Tehran, Iran.

Tel: +98 (912) 4266118

E-mail: rakhshankhah.md@gmail.com 
and acquiring normal levels of social and motor development in addition to the main existing symptoms [3]. The most prominent problem of ASD is disconnection from the social world and finally deficits in executive functions. Executive dysfunction has consistently been reported in individuals with ASDs [3]. Executive functions include a set of cognitive processes that involve distinct yet highly-organized components, such as cognitive flexibility, inhibitory control, attention, and working memory [4]. A main executive function is cognitive flexibility, i.e., related to switching attention between tasks, initiating new actions, and impulse control [5]; eventually, the regulations of thought and action [6]. Pan stated that exercise training could improve cognitive flexibility in children with ASDs [7].

In addition to cognitive dysfunction, motor actions seemed to be intact in children with ASDs; motor difficulties are among the other major problems of these children. Ozonoff et al. reported some special features in the gait pattern of children with ASDs, including shorter stride length, more flexed knees, abnormal upper trunk movements, and limited range of motion in elbows during gait [8]. Moreover, studies administered daily video and motion analysis of children with ASDs reported abnormal movements and delayed starting their motor actions [8, 9]. Interestingly, these abnormal movements have been reported to be associated with poor cognition functions [8, 9]. Moreover, the lack of bilateral coordination between hands and legs, balance problems, as well as delayed performance levels are other essential examples of motor difficulties in children with ASDs [10-12]. To explain the possible underlying mechanisms, Minshew et al. argued that maintaining and controlling an upright posture results from the integration and coherence of sensory inputs [13]. Children with ASDs experience deficits in central coherence and sensory integration; therefore, they encounter difficulties in maintaining and controlling an upright posture as well as motor coordination [13]. Moreover, Fournier et al. suggested that poor coordination observed in children with ASD is due to poor sensory integration of visual inputs [12]. Fundamental motor actions, such as locomotion, stabilization, and "give and take" exercises are associated with several sensory inputs or sensory stimulation [9, 14]. Subsequently, they may play an important role in improving and enhancing balance and motor ability. Motor dysfunction, as well as impaired cognitive flexibility, could lead to numerous physical and cognitive limitations for children; especially accomplishments in school and office and preventing inappropriate behaviors. In addition to restricting physi- cal activities and learning new motor skills, poor motor abilities in the long term could considerably affect the development of cognitive components. Thus, providing opportunities for children with ASD to engage in physical activity programs as well as improving their motor challenges seems necessary and effective. The current study aimed to survey the effects of the Sports, Play, and Active Recreation for Kids (SPARK) program on the motor and cognitive functions in children with ASDs. SPARK was planned by a multidisciplinary group to develop physical activity in schools [11]. Besides, it is considered simple, regular, and predictable play for children with ASDs. This exercise program can be useful for activities related to health-fitness and skill-fitness [3]. The main purpose of the present study was to investigate whether this exercise could improve motor skills (fine $\&$ gross motor skills) and cognitive flexibility in children with ASDs.

\section{Materials and Methods}

We assigned a convenience sample of 40 children with ASDs aged 5-12 years to this study from psychological clinics in Tehran and Qom Cities, Iran. The inclusion criteria for the participants were as follows: the study participants were supposed to be inspired enough to participate and tolerate a 45-minute exercise program and follow instructions and commands necessary for acceptable performance. The exclusion criteria were having medical contraindication (e.g. orthopedic or any acute conditions) to participate in the exercise programs. They were also excluded if they had a recent $(<4$ weeks) administration of any psychotropic medication. Patients and their families provided a written informed consent concerning the process and aim of the study prior to entering the study. The study protocol was approved by the Institutional Review Board at the University of Tehran and it was compliant with the Declaration of Helsinki.

The following measures were employed in the present research: Bruininks-Oseretsky test of motor proficiency (BOTMP): Bruininks provided the test using revising Oseretsky's motor skills test in 1978. BOTMP test is a valid instrument, including 8 subscales (53 separated parts) to assess motor functions, such as motor skills or fine and gross motor disorders in individuals aged $4-20$ years [15]. The abbreviated form of the test consists of 8 subscales, including 14 separated parts [16]. Four of the 8 subscales assess gross motor skills, 3 subscales assess fine motor skills, and the remaining one subscale evaluates gross and fine motor skills. The test-retest reliability of the BOTMP test has been reported as $87 \%$ [14]. 
For assessing executive function, we used the computer version of the Wisconsin Card Sorting Test (WCST) [7]. The WCST includes 4 stimulus cards and 128 response cards. One red triangle, 2 green stars, 3 yellow crosses, or 4 blue circles are demonstrated in the stimulus cards. The 128 response cards contain pictures combining different shapes (triangles, stars, crosses, \& circles), colors (red, blue, yellow, \& green), and numbers (1, 2, 3, or 4). The cards are presented on a screen in the computer version of the WCST. The participant has to choose an answer card for matching with one of the 4 key cards based on its potential characteristics with 4 cards.

The SPARK program involves 24 sessions (three 45min sessions per week) at an indoor gym. Four trained coaches with 2-year experience and an expert psychologist managed sessions based on 10-min warm-up activities, 25-min basic exercise, and finally 10-min cool-down activities. The SPARK discipline is based on National Association for Sport and Physical Education (NASPE) guidelines. A standard SPARK program has two parts; a health-fitness activity and a skill-fitness activity. Aerobic dance, running games, and jump ropes are related to health-fitness activities, such as cardiovascular endurance exercise and abdominal and upper body strength. The soccer, basketball, Frisbee games, football, and kickball that have the improving potential are examples of skill-fitness activities.

Children who met the eligibility criteria for the study enrollment entered the study. Following the initial assessment, the research participants were randomly separated into two groups of treatment and control $(n=20)$ group). The treatment group received SPARK programs three times per week for two months (24 sessions), including stability and locomotion exercises. Each session which started at 9 AM before lunchtime, lasted 30-45 minutes; the first and last 10 minutes of each session belonged to warm-up and cool-down states. The waitlist control group served the purpose of providing untreated participants and received their routine programs, except for the SPARK program. All study participants were assessed by the BOTMP test and WCST in three sessions (basic, before the start point, \& 2-7 days after the final session) by the same examiners.

We used SPSS for analyzing the collected data. Shapiro-Wilk test was used for examining the normality of the obtained data. To determine the influence of the intervention on test performance across the study groups, repeated-measures Analysis of Variance (ANOVA) and Analysis of Covariance (ANCOVA) were applied. The significance level was considered statistically $\mathrm{P}<0.05$.

\section{Results}

A total of 40 children with ASDs aged 5-12 years were assigned to the study. The treatment and control groups consisted of 20 participants each. Table 1 presents the descriptive data of the study participants. Table 2 indicates the mean scores of the BOTMP test at the baseline, pre-, and post-treatment in the research groups. The BOTMP test data indicated that in the total value of the motor skill subscale, there was a significant difference between the treatment and control groups in the discrepancy of the pretest, and posttest scores $(F=57.42, P=0.001)$. There were also significant differences in the fine motor skill ( $F=32.80, P=0.001)$, manual coordination $(\mathrm{F}=28.91, \mathrm{P}=0.009)$, body coordination ( $\mathrm{F}=43.28, \mathrm{P}=0.005)$, as well as strength and agility $(F=32.89 \mathrm{P}=0.001)$ subscales between the study groups. Moreover, as per Table 2, data analysis respecting cognitive flexibility measures indicated no significant differences in the total correct subscale $(F=19.84, P=0.53)$, perseverative responses ( $\mathrm{F}=14.21, \mathrm{P}=0.024)$, perseverative errors $(\mathrm{F}=19.98, \mathrm{P}=0.08)$, non-perseverative errors $(F=19.45, P=0.34)$, conceptual-level response $(F=21.47$, $\mathrm{P}=0.07)$, and categories completed $(\mathrm{F}=5.59, \mathrm{P}=0.06)$ between the treatment and control groups in the baseline, pre-test, and post-test scores.

\section{Discussion}

Our findings indicated that the motor skills of children with ASDs were affected by the SPARK. Children with ASDs encounter problems with fine and gross motor skills [17]. The current study results indicated that the SPARK has the potential to improve both types of motor skills in children and adolescents with ASDs. Moreover, our results were consistent with those of Yilmaz suggesting that 10-week swimming and aqua exercises could improve motor skills among 9-year-old children with ASDs [9]. The ability to control and maintain balance and perform exercise is a crucial element to successfully execute almost all daily physical activities. Researchers have highlighted the role of the complex interaction among skeletal, muscular, and nervous systems in controlling and maintaining the body for conducting exercises. Scholars have indicated that the importance of each system in controlling balance depends on the spatial situation as well as the purpose of the activity [18, 19]. In this model, the central nervous system makes use of proprioception, vestibular, and visual systems to be informed of the body's Center of Gravity (COG) relative to the body's Base of Support (BOS) through processing an appropriate response [20]. Furthermore, there also 
Table 1. Descriptive data of the study participants in both research groups

\begin{tabular}{ccccc}
\hline Group & No. & & Mean \pm SD \\
\cline { 3 - 5 } & & Age $(\mathbf{y})$ & Weight $(\mathbf{k g})$ & Height $(\mathbf{c m})$ \\
\hline Treatment & 20 & $8.08 \pm 2.06$ & $22.83 \pm 6.5$ & $120 \pm 1.07$ \\
Control & 20 & $7.14 \pm 2.23$ & $32.07 \pm 8.07$ & $122 \pm 1.5$ \\
\hline
\end{tabular}

Exercise Science and Medicine

Table 2. The Mean $\pm S D$ values of the BOTMP test and the WCST at the baseline, pretest, and posttest in the study groups

\begin{tabular}{|c|c|c|c|c|c|c|}
\hline \multirow{2}{*}{ Variables } & \multirow{2}{*}{ Group } & \multicolumn{3}{|c|}{ Mean $\pm S D$} & \multirow{2}{*}{$\mathbf{F}$} & \multirow{2}{*}{$\mathbf{P}$} \\
\hline & & Baseline & Pre-test & Post-test & & \\
\hline \multirow{3}{*}{ Fine motor skills } & Treatment & $45.00 \pm 11.64$ & $45.18 \pm 17.43$ & $50.55 \pm 12.60$ & \multirow{3}{*}{32.80} & \multirow{3}{*}{0.001} \\
\hline & & & & & & \\
\hline & Control & $45.36 \pm 11.94$ & $44.91 \pm 16.89$ & $45.64 \pm 17.70$ & & \\
\hline \multirow{3}{*}{ Manual coordination } & Treatment & $47.27 \pm 10.56$ & $48.00 \pm 11.83$ & $55.00 \pm 11.23$ & \multirow{3}{*}{28.91} & \multirow{3}{*}{0.009} \\
\hline & & & & & & \\
\hline & Control & $44.64 \pm 10.48$ & $43.09 \pm 11.32$ & $47.00 \pm 11.40$ & & \\
\hline \multirow{3}{*}{ Body coordination } & Treatment & $41.91 \pm 9.62$ & $42.91 \pm 10.63$ & $58.73 \pm 10.98$ & \multirow{3}{*}{43.28} & \multirow{3}{*}{0.005} \\
\hline & & & & & & \\
\hline & Control & $47.91 \pm 9.42$ & $48.00 \pm 8.75$ & $45.73 \pm 10.58$ & & \\
\hline \multirow{3}{*}{ Strength and agility } & Treatment & $67.36 \pm 5.99$ & $69.64 \pm 9.71$ & $73 \pm 9.47$ & \multirow{3}{*}{32.89} & \multirow{3}{*}{0.001} \\
\hline & & & & & & \\
\hline & Control & $65.36 \pm 11.75$ & $66.00 \pm 9.71$ & $66.27 \pm 8.33$ & & \\
\hline \multirow{3}{*}{ Total motor composite } & Treatment & $49.73 \pm 8.39$ & $60.09 \pm 10.11$ & $63.64 \pm 10.95$ & \multirow{3}{*}{57.42} & \multirow{3}{*}{0.001} \\
\hline & & & & & & \\
\hline & control & $48.48 \pm 8.51$ & $49.82 \pm 5.86$ & $61.55 \pm 8.61$ & & \\
\hline \multirow{3}{*}{ Total correct } & Treatment & $72.64 \pm 10.51$ & $81.73 \pm 9.37$ & $79.73 \pm 8.97$ & \multirow{3}{*}{19.84} & \multirow{3}{*}{0.53} \\
\hline & & & & & & \\
\hline & Control & $71.00 \pm 7.17$ & $72.09 \pm 6.12$ & $75.00 \pm 5.42$ & & \\
\hline \multirow{3}{*}{$\begin{array}{l}\text { Perseverative } \\
\text { responses }\end{array}$} & Treatment & $26.00 \pm 15.50$ & $15.36 \pm 12.58$ & $16.64 \pm 12.81$ & \multirow{3}{*}{14.21} & \multirow{3}{*}{0.24} \\
\hline & & & & & & \\
\hline & control & $18.55 \pm 9.26$ & $16.27 \pm 8.44$ & $12.82 \pm 9.50$ & & \\
\hline \multirow{5}{*}{ Non-perseverative } & Treatment & $22.36 \pm 17.81$ & $17.18 \pm 11.73$ & $11.91 \pm 11.55$ & \multirow{2}{*}{19.98} & \multirow{2}{*}{0.08} \\
\hline & control & $16.82 \pm 10.31$ & $12.63 \pm 10.28$ & $8.00 \pm 9.77$ & & \\
\hline & & & & & & \\
\hline & Treatment & $14.64 \pm 8.95$ & $12.45 \pm 8.63$ & $8.18 \pm 7.55$ & & \\
\hline & control & $15.36 \pm 7.55$ & $17.27 \pm 14.95$ & $6.00 \pm 3.61$ & & \\
\hline \multirow{2}{*}{$\begin{array}{l}\text { Conceptual-level } \\
\text { response }\end{array}$} & Treatment & $56.36 \pm 23.19$ & $66.55 \pm 10.42$ & $62.27 \pm 15.02$ & & \\
\hline & control & $55.45 \pm 9.83$ & $54.00 \pm 11.48$ & $74.09 \pm 7.42$ & 21.47 & 0.07 \\
\hline Categories com- & Treatment & $5.09 \pm 2.26$ & $4.18 \pm 1.60$ & $3.91 \pm 2.02$ & & \\
\hline pleted & control & $4.55 \pm 1.04$ & $6.41 \pm 1.26$ & $6.00 \pm 0.00$ & & \\
\hline
\end{tabular}


exist some other investigations, revealing that individuals who regularly perform physical activities or sports for a specific time indicated stronger ability for controlling the total body. For example, studies reported that walking regularly on an even surface-enhanced dynamic balance [21].

Besides, the current study results suggested that the SPARK also positively affected manual coordination, strength, and agility. This finding was consistent with Hodg's study [22]. However, the current outcome failed to support a previous finding demonstrating that coordination is strongly associated with the speed and precision of the action as well as fine and gross motor skills; generally, it results from a complicated motor ability. It is assumed that exercise has inadequate potential to make a significant change in improving coordination. Accordingly, McCleery et al. suggested that children with ASDs have poor cognitive and motor ability; therefore, this condition undoubtedly affects learning in this population. They also explained that the type of physical activity and exercise used also impact the acquisition of coordination following physical exercises [23, 24].

Unexpectedly, our findings revealed no change in executive function among the explored children with ASDs following the SPARK. This outcome was inconsistent with several previous findings; they indicated that physical exercises improved the cognitive flexibility of individuals with ASDs [25]. The authors had explained that increased cognitive function following a physical exercise is possibly due to tiredness, adequate motivation for motor actions, and the similarity between cognitive function and physical exercises. However, in the current study, fatigue could not influence stereotypical behaviors. This is because the posttest had been performed a week after the final session. Moreover, the SPARK exercises used in our study were not similar to cognitive flexibility behaviors in individuals with ASDs.

Courchesne et al. investigated the postmortem cerebellum of individuals with ASDs, aged 6-30 years [26]. The authors reported that in $95 \%$ of the samples, the cerebellum had no normal anatomical structure. Such studies indicated several deficits in the cerebellum of individuals with ASDs, including the lack of purkinje and granular neurons and deficits in the posterior hemisphere as well as vermis development [12]. Moreover, the vital role of the cerebellum in controlling motor actions, such as a fine and gross motor is well-evidenced and supported. Cerebellum serves as a bidirectional pathway between visual, auditory, and somatosensory cortices [12]. Levisohn et al. reported that the symp- toms and behavioral features of ASDs, including balance disorders, repetitive behaviors, and social isolation were observed [27]. Furthermore, Schmahmann et al. claimed that the main symptoms of the cerebellar cognitive-affective syndrome are balance disorders, problems in cognitive functions, problems in visuospatial memory, as well as behavioral challenges, including maladaptive social behaviors and communicating skills [28]. Qiu et al. also proposed that motor and social deficits are associated with the atypical structure of basal ganglia [29]. Therefore, a possible explanation for our findings is that the stability and locomotion exercises of the SPARK, as activities requiring learning skills, motor control, and social engagement, positively influence cortical, subcortical, and cerebellar functions. In the current study and other investigations, no direct measurement was considered for cerebellar function; however, the results of these studies support our interpretation. Thus, it is logical to attribute the prominent improvement of the social interaction of our participants to the improvement of cerebellar function following the SPARK.

However, there were some limitations in the current study that might limit the generalizability of the findings. First, the number of studied females was limited. Second, regarding the behavioral context of the intervention, it was impossible to ensure the blinding of the study participants. Finally, there were different motivation levels in our participants with ASDs and there was a variety in the extent to which they engaged in physical activities.

\section{Conclusion}

Generally, the selective SPARK exercises have the potential to enhance motor ability in children with ASDs. The SPARK could positively influence major motor limitations and challenge cognitive skills. Furthermore, these exercises are supposed to be conducted regularly to be effective in the long term.

\section{Ethical Considerations}

\section{Compliance with ethical guidelines}

There were no ethical considerations to be considered in this research.

\section{Funding}

The study received a financial support from the University of Tehran for this research (Grant Number: 30701.1.2). 


\section{Authors' contributions}

Conceptualization: Mahboubeh Ghayour Najafabadi; Writing - original draft: Sepideh Niyazi; Data coolection and Data analysis: Amir Sobrakhshan Khah.

\section{Conflict of interest}

The authors declared no conflict of interest.

\section{Acknowledgments}

The authors would like to acknowledge the financial support of the University of Tehran for this research (Grant Number: 30701.1.2).

\section{References}

[1] Ctr's for Disease Control and Prevention (CDC), United States of America. Revision of the Centers for Disease Control (CDC) surveillance case definition for acquired immunodeficiency syndrome. Morbidity and Mortality Weekly Report. 1987; 36:1S-15S. https:// www.cdc.gov/mmwr/pdf/other/mmsu3601.pdf

[2] Skodol AE, Gibbon M, Williams JBW, First MB. DSM-IV-TR casebook: A learning companion to the diagnostic and statistical manual of mental disorders. $4^{\text {th }}$ ed. Washington: American Psychiatric Publishing, Inc.; 2002. [DOI:10.1176/appi.books.9781585622665]

[3] Najafabadi MG, Sheikh M, Hemayattalab R, Memari A-H, Aderyani MR, Hafizi $S$. The effect of SPARK on social and motor skills of children with autism. Pediatrics \& Neonatology. 2018; 59(5):481-7. [DOI:10.1016/j.pedneo.2017.12.005] [PMID]

[4] Granader Y, Wallace GL, Hardy KK, Yerys BE, Lawson RA, Rosentha $M$, et al. Characterizing the factor structure of parent reported executive function in Autism Spectrum Disorders: The impact of cognitive inflexibility. Journal of Autism and Developmental Disorders. 2014; 44(12):3056-62. [DOI:10.1007/s10803-014-2169-8] [PMID] [PMCID]

[5] de Andrade Varanda C, Fernandes FDM. Assessing cognitive flexibility, communication, social interaction and interest patterns of persons with autism as a basis for intervention. Psychology. 2015; 6(04):387. [DOI:10.4236/psych.2015.64035]

[6] Becker DR, Miao A, Duncan R, McClelland MM. Behavioral self-regulation and executive function both predict visuomotor skills and early academic achievement. Early Childhood Research Quarterly. 2014; 29(4):411-24. [DOI:10.1016/j.ecresq.2014.04.014]

[7] Pan C-Y, Chu C-H, Tsai C-L, Sung M-C, Huang C-Y, Ma W-Y. The impacts of physical activity intervention on physical and cognitive outcomes in children with Autism Spectrum Disorder. Autism. 2017; 21(2):190-202. [DOI:10.1177/1362361316633562] [PMID]

[8] Ozonoff S, Young GS, Goldring S, Greiss-Hess L, Herrera AM, Steele J, et al. Gross motor development, movement abnormalities, and early identification of autism. Journal of Autism and Developmental Disorders. 2008; 38(4):644-56. [DOI:10.1007/s10803-007-0430-0] [PMID] [PMCID]
[9] Yilmaz I, Yanardağ M, Birkan B, Bumin G. Effects of swimming training on physical fitness and water orientation in autism. Pediatrics International. 2004; 46(5):624-6. [DOI:10.1111/j.1442 200x.2004.01938.x] [PMID]

[10] Tyler K, MacDonald M, Menear K. Physical activity and physica fitness of school-aged children and youth with Autism Spectrum Disorders. Autism Research and Treatment. 2014; 2014:312163. [DOI:10.1155/2014/312163] [PMID] [PMCID]

[11] Jansiewicz EM, Goldberg MC, Newschaffer CJ, Denckla MB Landa R, Mostofsky SH. Motor signs distinguish children with high functioning autism and Asperger's syndrome from controls. Journal of Autism and Developmental Disorders. 2006; 36(5):613-21. [DOI:10.1007/s10803-006-0109-y] [PMID]

[12] Fournier KA, Hass CJ, Naik SK, Lodha N, Cauraugh JH. Motor coordination in Autism Spectrum Disorders: A synthesis and metaanalysis. Journal of Autism and Developmental Disorders. 2010 40(10):1227-40. [DOI:10.1007/s10803-010-0981-3] [PMID]

[13] Minshew NJ, Sung K, Jones BL, Furman JM. Underdevelopment of the postural control system in autism. Neurology. 2004; 63(11):205661. [DOI:10.1212/01.WNL.0000145771.98657.62] [PMID]

[14] Fisher BE, Wu AD, Salem GJ, Song J, Lin C-HJ, Yip J, et al. The effect of exercise training in improving motor performance and corticomotor excitability in people with early Parkinson's disease. Archives of Physical Medicine and Rehabilitation. 2008; 89(7):1221-9. [DOI:10.1016/j.apmr.2008.01.013] [PMID] [PMCID]

[15] South M, Palilla J. Bruininks-Oseretsky test of motor proficiency. Encyclopedia of Autism Spectrum Disorders. Berlin: Springer; 2013. https://books.google.com/books/about/Encyclopedia_of_Autism Spectrum_Disorder.html?id=E8QDtwAACAAJ

[16] Lucas BR, Latimer J, Doney R, Ferreira ML, Adams R, Hawkes G, et al. The Bruininks-Oseretsky test of motor proficiency-short form is reliable in children living in remote Australian aboriginal communities. BMC Pediatrics. 2013; 13(1):135. [DOI:10.1186/1471-2431-13135] [PMID] [PMCID]

[17] Fournier KA, Kimberg Cl, Radonovich KJ, Tillman MD, Chow JW Lewis $\mathrm{MH}$, et al. Decreased static and dynamic postural control in children with Autism Spectrum Disorders. Gait \& Posture. 2010; 32(1):6-9. [DOI:10.1016/j.gaitpost.2010.02.007] [PMID] [PMCID]

[18] Cuccia A, Caradonna C. The relationship between the stomatognathic system and body posture. Clinics. 2009; 64(1):61-6. [DOI:10.1590/S1807-59322009000100011] [PMID] [PMCID]

[19] Sherwood L. Human physiology: From cells to systems. Boston: Cengage Learning; 2015. https://books.google.com/books/about/Human_Physiology_From_Cells_to_Systems.html?id=8WVvCgAAQBAJ

[20] Marsh DW, Richard LA, Williams AL, Lynch KJ. The relationship between balance and pitching error in college baseball pitchers. The Journal of Strength \& Conditioning Research. 2004; 18(3):441-6. [DOI:10.1519/R-13433.1]

[21] Stöggl T, Haudum A, Birklbauer J, Murrer M, Müller E. Short and long term adaptation of variability during walking using unstable (Mbt) shoes. Clinical Biomechanics. 2010; 25(8):816-22. [DOI:10.1016/j.clinbiomech.2010.05.012] [PMID]

[22] Crowe TK, Horak FB. Motor proficiency associated with vestibular deficits in children with hearing impairments. Physical Therapy. 1988; 68(10):1493-9. [DOI:10.1093/ptj/68.10.1493] 
[23] Schunk DH. Self-regulated learning: The educational legacy of Paul R. Pintrich. Educational Psychologist. 2005; 40(2):85-94. [DOI:10.1207/s15326985ep4002_3]

[24] McCleery JP, Elliott NA, Sampanis DS, Stefanidou CA. Motor development and motor resonance difficulties in autism: Relevance to early intervention for language and communication skills. Frontiers in Integrative Neuroscience. 2013; 7:30. [DOI:10.3389/fnint.2013.00030] [PMID] [PMCID]

[25] Lang R, Koegel LK, Ashbaugh K, Regester A, Ence W, Smith W. Physical exercise and individuals with Autism Spectrum Disorders: A systematic review. Research in Autism Spectrum Disorders. 2010; 4(4):565-76. [DOI:10.1016/j.rasd.2010.01.006]

[26] Courchesne E, Yeung-Courchesne R, Press GA, Hesselink JR, Jernigan TL. Hypoplasia of cerebellar vermal lobules VI and VII in autism. New England Journal of Medicine. 1988; 318:1349-54. [DOI:10.1056/NEJM198805263182102] [PMID]

[27] Levisohn L, Cronin-Golomb A, Schmahmann JD. Neuropsychological consequences of cerebellar tumour resection in children Cerebellar cognitive affective syndrome in a paediatric population. Brain. 2000; 123(5):1041-50. [DOI:10.1093/brain/123.5.1041] [PMID]

[28] Schmahmann JD, Sherman JC. The cerebellar cognitive affective syndrome. Brain. 1998; 121(4):561-79. [DOI:10.1093/ brain/121.4.561] [PMID]

[29] Qiu A, Adler M, Crocetti D, Miller MI, Mostofsky SH. Basal ganglia shapes predict social, communication, and motor dysfunctions in boys with Autism Spectrum Disorder. Journal of the American Academy of Child \& Adolescent Psychiatry. 2010; 49(6):539-51.e4 [DOI:10.1016/j.jaac.2010.02.012] [PMID] 
This Page Intentionally Left Blank 